\title{
Serum Markers of Cardiac Complications In a Systemic Sclerosis Cohort
}

\section{Anders Heiervang Tennøe}

Oslo University Hospital

\section{Klaus Murbræch}

Oslo University Hospital

Henriette Didriksen

Oslo University Hospital

\section{Thor Ueland}

Oslo University Hospital

Vyacheslav Palchevskiy

University of California, Los Angeles

\section{Stephen Weigt}

University of California, Los Angeles

Håvard Fretheim

Oslo University Hospital

\section{Øyvind Midtvedt}

Oslo University Hospital

\section{Torhild Garen}

Oslo University Hospital

\section{Cathrine Brunborg}

Oslo University Hospital

\section{Pål Aukrust}

University of Oslo

\section{Øyvind Molberg}

Oslo University Hospital

John Belperio

University of California, Los Angeles

Anna-Maria Hoffmann-Vold ( $\square$ a.m.hoffmann-vold@medisin.uio.no )

Oslo University Hospital

\section{Research Article}


Keywords: Systemic sclerosis, Cardiac disease, Serological biomarkers, Echocardiography, Systolic dysfunction, Diastolic dysfunction

Posted Date: December 17th, 2021

DOI: https://doi.org/10.21203/rs.3.rs-1141010/v1

License: (c) (i) This work is licensed under a Creative Commons Attribution 4.0 International License. Read Full License

Version of Record: A version of this preprint was published at Scientific Reports on March 18th, 2022. See the published version at https://doi.org/10.1038/s41598-022-08815-8. 


\section{Abstract}

Background: Primary cardiac involvement is one of the leading causes of mortality in systemic sclerosis (SSc), but little is known regarding circulating biomarkers for cardiac SSc. Here, we aimed to investigate potential associations between cardiac SSc and candidate serum markers.

Methods: Serum samples from patients of the Oslo University SSc cohort and 100 healthy controls were screened against two custom-made candidate marker panels containing molecules deemed relevant for cardiopulmonary and/or fibrotic diseases. Left (LV) and right ventricular (RV) dysfunction was assessed by protocol echocardiography, performed within three years from serum sampling. Patients suspected of pulmonary hypertension underwent right heart catheterization. Vital status at study end was available for all patients. Descriptive analyses, logistic and Cox regressions were conducted to assess associations between cardiac SSc and candidate serum markers.

Results: The 371 patients presented an average age of $57.2( \pm 13.9)$ years. Female sex $(84 \%)$ and limited cutaneous SSc (73\%) were predominant. Association between LV diastolic dysfunction and tumor necrosis factor-related apoptosis-inducing ligand (TRAIL) (OR $0.41,95 \% \mathrm{Cl} 0.21-0.78, p=0.007$ ) was identified. LV systolic dysfunction defined by global longitudinal strain was associated with angiopoietin 2 (ANGPT2) (OR 3.42, 95\% Cl 1.52-7.71, p=0.003) and osteopontin (OPN) (OR 1.95, 95\% Cl 1.08-3.52, $\mathrm{p}=0.026)$. RV systolic dysfunction, measured by tricuspid annular plane systolic excursion, was associated to markers of LV dysfunction (ANGPT2, OPN, and TRAIL) (OR 1.67, 95\% Cl 1.11-2.50, $\mathrm{p}=0.014$, OR $1.86,95 \% \mathrm{Cl} 1.25-2.77, \mathrm{p}=0.002$, OR $0.32,95 \% \mathrm{Cl} 0.15-0.66, \mathrm{p}=0.002$, respectively) and endostatin (OR $1.86,95 \% \mathrm{Cl} 1.22-2.84, \mathrm{p}=0.004)$.

Conclusion: ANGPT2, OPN and TRAIL seem to be circulating biomarkers associated with both LV and RV dysfunction in SSc.

\section{Key Messages}

- Angiopoietin 2, osteopontin and tumor necrosis factor-related apoptosis-inducing ligand are circulating biomarkers associated with both left and right ventricular dysfunction in SSc.

- Endostatin is associated with right ventricular systolic dysfunction in SSc.

- Angiopoietin 2, osteopontin and tumor necrosis factor-related apoptosis-inducing ligand are strong independent predictors of mortality in SSc.

\section{Introduction}

Systemic sclerosis (SSc) is a multi-organ, progressive disorder hallmarked by dysregulation of the immune system, vasculopathy and target organ fibrosis $(1,2)$. The heart is frequently affected in SSc and is together with lung involvement reported to account for the majority of disease-related causes of deaths (3-6). The pro-fibrotic pathophysiology in SSc is still inadequately understood (1). Cardiac fibrosis in SSc 
is thought to be related to repeated focal ischaemia leading to irreversible lesions and/or inflammatory myocarditis, but these issues have not been fully elucidated $(7,8)$. From our recent studies and others, we know that left and right ventricular (LV and RV) systolic dysfunction, as well as LV diastolic dysfunction, are common complications of SSc (9-11). LV diastolic dysfunction further progresses over time and displays a poor prognosis (9). We have also presented multivariable models predicting mortality in SSc $(9,10)$. Such models may be improved by additional predictor variables as knowledge of SSc pathology improves. Early detection of these cardiac complications is believed beneficial to improve outcome $(9$, 10). However, there exist no established guidelines for screening of cardiac involvement as for pulmonary arterial hypertension (PAH) where screening with annual echocardiography is recommended $(12,13)$.

The structure-function data provided by echocardiography is highly useful to diagnose established cardiac dysfunctions. However, to identify SSc patients at risk of developing, or progression of cardiac dysfunction, there is a need for easily accessible algorithms including biological markers. The detection of SSc associated pulmonary hypertension $(\mathrm{PH})$ is based on clinical, imaging, but also serological markers (14). N-terminal pro-brain natriuretic peptide (NT-proBNP) is a highly valued serological marker of general cardiac dysfunction (15). However, apart from the serum autoantibody anti-topoisomerase I (ATA) and NT-proBNP, there exist to our knowledge limited data on serological markers associated with cardiac dysfunction in SSc (16-18).

Biomarkers are a prioritized research area in SSc as they may bring information regarding the disease itself and/or disease-related organ afflictions. Biomarkers may be divided into three categories: i) diagnostic, ii) prognostic for disease extent and severity and iii) predictive for distinct organ complications and/or responsiveness to therapeutic intervention $(19,20)$. In SSc, we have previously shown that the homeostatic chemokine CCL21 and the anti-angiogenic molecule endostatin are associated with, and predict, new onset PAH (21-23). Other studies have described additional markers with diagnostic and/or prognostic potential in other pro-fibrotic disorders with cardiopulmonary complications, justifying an evaluation in cardiac SSc. In example, the extracellular matrix protein osteopontin (OPN) is reported related to cardiac fibrosis in mice (24) and tumor necrosis factor-related apoptosis-inducing ligand (TRAIL) has been associated with PH in humans and rodents $(25,26)$. Finally, as the interplay between fibrosis and immunological dysregulation in SSc is poorly understood, there is hope that investigation of associations between the disease and circulating serum markers linked to profibrotic pathways may give clues to underlying pathophysiologic mechanisms and potentially novel treatment approaches.

In the present study, we assessed relations between two custom-made serum marker panels containing molecules deemed relevant for cardiac, pulmonary and/or fibrotic diseases and early stage SSc as a first proof of concept study. Biomarkers related to SSc were further evaluated for diagnostic and predictive abilities for cardiac dysfunction and mortality, respectively.

\section{Materials And Methods}




\subsection{SSc study cohort}

All SSc patients seen at the department of rheumatology at Oslo University Hospital are included in the prospective Oslo SSc cohort, and are followed on an annual basis (21). At first visit, patients undergo clinical examination (including assessment of digital ulcers, calcinosis and modified Rodnan skin score), serum sampling (including anti-centromere (ACA) and anti-topoisomerase-I (ATA) antibody status), pulmonary function tests, high-resolution CT (HRCT) scan and echocardiography (9). Annual follow-up consultations are performed by a rheumatologist, registering data on demographic, clinical, laboratory, functional and imaging parameters in the Norwegian Systemic Connective Tissue Disease and Vasculitis Registry (NOSVAR) (21).

This study included patients presenting at our hospital from 2002-2016. Inclusion criteria for this study were (i) fulfillment of the 1980 American College of Rheumatology criteria for SSc (27) and/or the 2013 European League Against Rheumatism/American College of Rheumatology criteria (28), (ii) age > 18 years and (iii) serum samples available for serum marker analysis. Demographic, clinical and laboratory data were extracted from NOSVAR and electronic patient journals (EPJs). Patients were classified as limited (IcSSc) or diffuse cutaneous (dcSSc) SSc according to skin involvement (29). Data on medication were collected from EPJs and registered if ever used. Vital status was available for all patients at study end, extracted from the Norwegian national registry. Disease duration was defined as time from SSc diagnosis to serum sampling and observation period as time from serum sampling to death or study end. The study was approved by The Regional Committee of Health and Medical Research Ethics in SouthEast Norway, research protocols no. 2016/119 and 2017/1815. All included participants consented to serum sampling for research purposes. All methods were performed in accordance with the relevant guidelines and regulations.

\subsection{Serum sampling and biomarker analyses}

Serum samples are collected from all SSc patients entering NOSVAR at first visit (baseline) and annual visits, using a standardized protocol for analyzation following the European Scleroderma Trial and Research (EUSTAR) recommendations for biobanking (30). For this study, baseline sera were used. Serum was centrifuged within 30 minutes at room temperature and stored at $-70{ }^{\circ} \mathrm{C}$ before analyzation. Two custom-made panels were applied including specific circulating markers tailored for cardiac and pulmonary disease based on literature review and expert opinion through author discussion. Samples from 2003-2016 were in 2016 analyzed by Luminex (panel A), using Milliplex assays (Merck Millipore), evaluating markers known from immune-related and pro-fibrotic disease states $(31,32)$. In 2013, serum samples collected from 2002-2013 were analyzed by enzyme-linked immunosorbent assay (ELISA) (panel B) (R\&D Systems) for serum markers known to associate with cardiac or pulmonary disease (21, 33). All serological markers of Panel A and B are listed in Supplementary Table 2. One hundred randomly selected blood bank donors served as healthy controls, solely regarding serum marker sampling, and were analyzed in both steps. According to Norwegian law, only individuals without infection, cardiovascular disease, immunodeficiency or any chronic disease are allowed to donate blood (21). 


\subsection{Cardiopulmonary assessment}

We had comprehensive cardiac assessments available. Systolic and diastolic cardiac function were evaluated by one of the authors (AHT) on available echocardiographies, applying updated international recommendations as previously shown $(9,34)$. Systolic dysfunction was defined by a GLS $>-17.0 \%(10$, $35)$, rather than ejection fraction, due to better reproducibility. LV diastolic dysfunction was evaluated according to the 2016 guidelines as described earlier $(9,36)$ and RV systolic dysfunction was defined by tricuspid annular plane systolic excursion (TAPSE) $<17 \mathrm{~mm}(10,34)$.

Suspicion of $\mathrm{PH}$ was based on patient history, symptoms/findings of $\mathrm{PH}$, echocardiography findings, or the DETECT algorithm $(14,37)$, warranting referral to right heart catheterization (RHC) for PH diagnosis. According to recent suggestions from the $6^{\text {th }}$ World Symposium for $\mathrm{PH}$, precapillary $\mathrm{PH}$ was defined as mean pulmonary artery pressure (mPAP) $\geq 21 \mathrm{~mm} \mathrm{Hg}$, pulmonary arterial wedge pressure $\leq 15 \mathrm{~mm} \mathrm{Hg}$ and pulmonary vascular resistance (PVR) $\geq 3.0$ Wood units (38). PAH and PH associated with ILD (PHILD) were defined as precapillary PH in absence or presence of ILD, respectively (37). ILD was expressed as extent of ILD in percentage of total lung volumes on HRCT. Significant ILD was defined as presence of ILD affecting $>10 \%$ of pulmonary tissue $(4,39)$. Extent of ILD on HRCT of $0.1-10 \%$ was defined mild ILD (4).

\subsection{Outcome measures}

As this was the first proof of concept study assessing circulating biomarkers in cardiac dysfunction in SSc, we aimed to first assess sera levels in SSc compared to healthy controls. In a second step, we tested associations between biomarkers and cardiac dysfunction. Lastly, we assessed biomarker predictive ability for all-cause mortality. Outcome measures were as follows:

1. Serum levels of the respective markers in SSc patients compared to values of healthy controls. Markers showing significant up- or downregulated median values in clinical SSc subsets, compared to controls, were further analyzed for association with cardiac dysfunction.

2. Association between serum biomarkers and the presence of (i) LV systolic dysfunction, assessed by GLS (ii) $L V$ diastolic dysfunction assessed by e', E/e', left atrial volume index and tricuspid regurgitant velocity and (iii) $R V$ systolic dysfunction assessed by tricuspid annular plane systolic excursion (TAPSE) were determined in all cases with conducted echocardiography within three years of blood sampling.

3. Serum markers showing association with echocardiographic dysfunction were tested for mortality predictive ability.

\subsection{Statistical analyses:}

Statistical analyses were performed using STATA version 16 (StataCorp LLC, College Station, Texas, USA) and SPSS version 25 (IBM, Armonk, New York, USA). Independent t-tests and chi square tests were 
applied for between-group comparisons as appropriate to assess differences in sera levels between SSc patients and healthy controls.

Candidate predictors for logistic and cox regression analyses were selected by expert opinion and based on the published literature $(21,31-33)$. Logistic regressions with odds ratios (OR) and $95 \%$ confidence intervals $(\mathrm{Cl})$ were applied for evaluation of association between cardiac outcomes and serological biomarkers. Parameters with significance levels $<0.20$ were included in multivariable models adjusting for age and sex. Multivariable logistic regression analyses were preceded by correlation tests in order to avoid multicollinearity. In multivariable models, significance levels $<0.05$ were considered significant. Multivariable models were evaluated by area under the receiver-operating curve (AUC), and models presenting values $>0.7$ were considered acceptable and reported. Schoenfeld's test was applied for affirmation of proportional hazards. For confirmation of clinical significance within the SSc cohort, serum

markers associated with cardiac dysfunction were tested for mortality predicting abilities, adding them to multivariable models including diastolic dysfunction (model A) and precapillary PH (model B), as reported earlier (9). These reported models included clinical-, imaging- and serological markers, and proved superior predictive ability compared to models with other appreciated risk factors, such as e.g. presence of ischemic heart disease. Cox regressions with hazard ratios (HR) and $95 \% \mathrm{Cl}$ were calculated for the predictive value of serum biomarkers on mortality. C-statistics were applied to compare multivariable Cox models, with c-indexes $>0.7$ representing acceptable models.

\section{Results}

\subsection{Study cohort and serum markers associating with SSc and selected disease features}

The study cohort included 371 SSc patients with serum samples that could be analyzed for markers of panel A containing immune-related molecules and other proteins linked to pro-fibrotic and inflammatory pathways. Among these, 255 patients (69\%) had serum samples also analyzed for markers of panel B, enriched for molecules previously shown to associate with human cardiac or pulmonary diseases (40, 41). There were no differences between patient characteristics and demographics with or without panel $B$ evaluation with respect to age, sex, subtype or autoantibodies. Most patients were female $(n=313,84 \%)$ and presented with IcSSc ( $n=269,73 \%)$. Average age at serum sampling for panel A was $57 \pm 14$ years and median disease duration was 2.5 years (IQR 0.7-8.1). Demographic and serological features are shown in Table 1. Data on cardiovascular medications are shown in Supplementary Table 1. Seventy-four patients presented with precapillary PH (46 with PAH and 28 with PH-ILD). To assess the first outcome measure, serum levels of the individual panel A and B markers were compared between the SSc patients and healthy controls. Markers with significantly altered serum levels in SSc patients compared to controls are displayed in Figure 1. We identified altered levels of 26 serum markers in SSc with varying levels in dcSSc, and the two major SSc specific autoantibodies, ACA and ATA (Figure 1).

Table 1: Systemic sclerosis specific demographics and cardiac outcome measures of patients evaluated by panel $\mathrm{A}$. 


\begin{tabular}{|c|c|c|}
\hline & $\begin{array}{l}\text { Patients with available data, } \\
\mathrm{n}(\%)\end{array}$ & Panel A \\
\hline Age at serum sampling, years & $364 / 371$ (98) & $57.2(13.9)$ \\
\hline Female, n (\%) & $371 / 371(100)$ & $313(84)$ \\
\hline Disease duration, years & $364 / 371$ (98) & $2.5(0.7-8.1)$ \\
\hline Observation period, years & $363 / 371$ (98) & $5.9(3.1-8.3)$ \\
\hline Mortality, n (\%) & $371 / 371(100)$ & $103 / 371(28)$ \\
\hline IcSSc, n (\%) & $362 / 371$ (98) & $269(73)$ \\
\hline ACA, n (\%) & $370 / 371$ (99) & $196(53)$ \\
\hline ATA, n (\%) & $370 / 371$ (99) & $58(16)$ \\
\hline mRSS & $355 / 371(96)$ & $5(2-13)$ \\
\hline $\mathrm{BMI}, \mathrm{kg} / \mathrm{m}^{2}$ & $243 / 371(65)$ & $24(4)$ \\
\hline ILD, > $10 \%$ fibrosis & $356 / 371(96)$ & $87(24)$ \\
\hline Smoking & 234/371 (63) & $135(58)$ \\
\hline Hypertension & $246 / 371(66)$ & $31(13)$ \\
\hline Ischemic heart disease & 246/371 (66) & $42(17)$ \\
\hline GLS > -17.0\%, n (\%) & 111/371 (30) & $30(27)$ \\
\hline Diastolic dysfunction, n (\%) & 135/371 (36) & $37(27)$ \\
\hline TAPSE < 17 mm, n (\%) & 167/371 (45) & $29(17)$ \\
\hline
\end{tabular}

Data are presented as mean (SD), median (IQR) or number (percentage). ACA, anti-centromere antibodies; ATA, anti-topoisomerase I antibodies; BMI, body mass index; GLS, global longitudinal strain; ILD, interstitial lung disease; IcSSc, limited cutaneous systemic sclerosis; mRSS, modified Rodnan skin score; TAPSE, tricuspid annular plane systolic excursion. Parameters of cardiac function are evaluated on echocardiographies performed within three years from blood sampling.

\subsection{Patients evaluated for cardiac dysfunction in the study cohort}

Echocardiographic data on GLS, LV diastolic dysfunction and/or TAPSE, within three years from serum sampling, was available in 188/371 (51\%) study cohort patients (Figure 2). Patients with available echocardiography within three years from serum sampling were older than the patients without available echocardiography ( 60.0 vs 54.2 years, $p<0.001$ ), they had a shorter observation period (4.9 vs 7.5 years, $p<0.001)$ and higher mortality (38\% vs $17 \%, p<0.001)$. There were no differences with respect to sex, disease subtype, antibodies (ATA and ACA) or disease duration at serum sampling. 
To assess the second outcome, association of circulating biomarkers and cardiac dysfunction including $L V$ systolic dysfunction, LV diastolic dysfunction and RV systolic dysfunction in SSc, we analyzed all patients with available sera and echocardiographic data.

LV systolic dysfunction assessed by GLS was available in 111/188 (59 \%), of whom 30/111 (27 \%) had systolic dysfunction defined as GLS>-17.0\% (Figure 2). Compared to patients with normal GLS, the patients with low GLS were more often male ( $43 \%$ vs $11 \%, p<0.001$ ), but did not differ regarding age, subtype, ACA, ATA, precapillary PH, disease duration or observation period. By adjusting for age and sex in logistic regression, systolic dysfunction by GLS showed significant association to two of the diseaselinked serum markers displayed in Figure 1; angiopoietin 2 (ANGPT2) and OPN (Table 2).

Table 2: Association between echocardiographic ventricular dysfunction and serum markers adjusted for age and sex.

\begin{tabular}{|c|c|c|c|}
\hline Serum marker & OR & $95 \% \mathrm{Cl}$ & p-value \\
\hline \multicolumn{4}{|c|}{ Left ventricular systolic dysfunction, GLS >17\% } \\
\hline ANGPT2 $(n=100)$ & 3.42 & $1.52-7.71$ & 0.003 \\
\hline OPN $(n=93)$ & 1.95 & $1.08-3.52$ & 0.026 \\
\hline \multicolumn{4}{|c|}{ Left ventricular diastolic dysfunction } \\
\hline $\operatorname{ANGPT2}(n=122) *$ & 1.38 & $0.98-1.94$ & $0.062^{*}$ \\
\hline Endostatin $(n=113) *$ & 1.60 & $0.95-2.67$ & $0.075^{\star}$ \\
\hline TRAIL $(n=131)$ & 0.41 & $0.21-0.78$ & 0.007 \\
\hline \multicolumn{4}{|c|}{ Right ventricular systolic dysfunction, TAPSE $<17 \mathrm{~mm}$} \\
\hline ANGPT2 $(n=153)$ & 1.67 & $1.11-2.50$ & 0.014 \\
\hline Endostatin $(n=143)$ & 1.86 & $1.22-2.84$ & 0.004 \\
\hline OPN $(n=143)$ & 1.86 & $1.25-2.77$ & 0.002 \\
\hline TRAIL $(n=163)$ & 0.32 & $0.15-0.66$ & 0.002 \\
\hline
\end{tabular}

ANGPT2, angiopoietin 2; Cl, confidence interval; GLS, global longitudinal strain; OPN, osteopontin; OR, odds ratio; TAPSE, tricuspid annular plane systolic excursion; TRAIL, tumor necrosis factor-related apoptosis-inducing ligand. *indicates numerical associations. HR of ANGPT2, Endostatin, OPN and TRAIL represent an increase of one standard deviation.

3.4 Serum markers associated with left ventricular diastolic dysfunction 
Echocardiographic evaluation of LV diastolic dysfunction by e', E/e', left atrial volume index and tricuspid regurgitant velocity according to recommendations, was performed within three years of serum sampling in 135 of the study cohort patients with available echo (72\%). Among these, 37/135 (27\%) presented with diastolic dysfunction. There were no differences between patients segregated by diastolic function with respect to disease duration, sex, SSc subtype, ACA or ATA. Patients with diastolic dysfunction had higher age (64.6 vs 55.8 years, $p<0.001$ ), more frequent coexisting precapillary $\mathrm{PH}(67 \%$ vs $21 \%$, $p<0.001)$ and shorter observation period $(2.6$ vs 6.1 years, $p<0.001)$ than patients with normal diastolic function. By adjusting for age and sex in logistic regression, diastolic dysfunction was associated with TRAIL, while showing numerical associations with ANGPT2 and endostatin (Table 2).

\subsection{Serum markers associated with RV systolic dysfunction}

RV systolic dysfunction was assessed by TAPSE in 167/188 patients ( $89 \%$ ), with TAPSE $<17 \mathrm{~mm}$, indicating RV systolic dysfunction, present in 29/167 patients (17\%). Patients with low TAPSE presented numerical higher age (63.1 vs 58.3 years, $\mathrm{p}=0.074$ ), more frequent precapillary $\mathrm{PH}$ during the observation period ( $64 \%$ vs $30 \%, p=0.001)$ and shorter observation period ( 2.8 vs 5.3 years, $p<0.001)$ than patients with normal TAPSE. There were no differences between patients segregated by low/normal TAPSE with respect to disease duration, sex, SSc subtype, ACA or ATA. In multivariable logistic regression adjusting for age and sex, RV systolic dysfunction was independently associated with ANGPT2, endostatin, OPN and TRAIL.

Sera levels of ANGPT2, OPN and TRAIL of SSc patients compared to healthy controls are shown in Supplementary Figure 1.

\subsection{Serum markers associated with mortality}

Over the observation period of median 5.9 years (IQR 3.1-8.3), 103/371 patients (28 \%) deceased. Patients who died were older (65.5 vs 53.9 years, $p<0.001$ ), had longer disease duration at serum sampling ( 3.9 vs 2.0 years, $p=0.004$ ), shorter observation period ( 2.6 vs 7.5 years, $p<0.001)$, were more often male $(22.3 \%$ vs $13.1 \%, p=0.028)$, and presented more often dcSSc (34.3\% vs $22.3 \%, p=0.019)$, compared to survivors.

In our previous publication, we have presented two multivariable prediction models for SSc mortality (9). When we added serological markers separately to Model A (including diastolic dysfunction, sex, age at echocardiography, RV systolic function, diffusion capacity of the lungs, NT-proBNP and extent of skin affliction), ANGPT-2 and OPN remained independent predictors of mortality in their respective models (Table 3), yet excluding NT-proBNP from Model A due to insignificant $p$-value. When we added cardiac markers separately to Model B (including precapillary PH, sex, age at echocardiography, NT-proBNP and extent of skin affliction), ANGPT-2 and TRAIL remained independent predictors of mortality, retaining significance for all parameters of the original model (Table 4).

Table 3: Prediction of mortality by angiopoietin 2 and OPN in multivariable cox regression. 


\begin{tabular}{|c|c|c|c|c|c|c|c|}
\hline \multicolumn{8}{|c|}{ Multivariable cox regression on mortality } \\
\hline & $\mathrm{HR}$ & $95 \% \mathrm{Cl}$ & $\begin{array}{l}\mathrm{p}- \\
\text { value }\end{array}$ & & $\mathrm{HR}$ & $95 \% \mathrm{Cl}$ & $\begin{array}{l}\mathrm{p}- \\
\text { value }\end{array}$ \\
\hline ANGPT2 & 1.52 & $\begin{array}{l}1.20- \\
1.93\end{array}$ & 0.001 & OPN & 1.39 & $\begin{array}{l}1.01- \\
1.92\end{array}$ & 0.044 \\
\hline Age & 1.04 & $\begin{array}{l}1.01- \\
1.08\end{array}$ & 0.024 & Age & 1.05 & $\begin{array}{l}1.01- \\
1.09\end{array}$ & 0.023 \\
\hline $\begin{array}{l}\text { Diastolic } \\
\text { dysfunction }\end{array}$ & 3.71 & $\begin{array}{l}1.62- \\
8.48\end{array}$ & 0.002 & $\begin{array}{l}\text { Diastolic } \\
\text { dysfunction }\end{array}$ & 3.73 & $\begin{array}{l}1.76- \\
7.90\end{array}$ & 0.001 \\
\hline DLCO, \% & 0.95 & $\begin{array}{l}0.93- \\
0.97\end{array}$ & $<0.001$ & DLCO, \% & 0.95 & $\begin{array}{l}0.93- \\
0.97\end{array}$ & $<0.001$ \\
\hline Male sex & 0.94 & $\begin{array}{l}0.37- \\
2.36\end{array}$ & 0.888 & Male sex & 0.79 & $\begin{array}{l}0.30- \\
2.11\end{array}$ & 0.638 \\
\hline mRSS & 1.07 & $\begin{array}{l}1.03- \\
1.11\end{array}$ & $<0.001$ & mRSS & 1.05 & $\begin{array}{l}1.01- \\
1.08\end{array}$ & 0.006 \\
\hline TAPSE & 0.32 & $\begin{array}{l}0.13- \\
0.83\end{array}$ & 0.018 & TAPSE & 0.26 & $\begin{array}{l}0.12- \\
0.57\end{array}$ & 0.001 \\
\hline C-index & 0.87 & & & C-index & 0.89 & & \\
\hline
\end{tabular}

ANGPT2, angiopoietin 2; Cl, confidence interval; DLCO, diffusion capacity of the lungs for carbon monoxide; HR, hazard ratio; mRSS, modified Rodnan skin score; OPN, osteopontin; TAPSE, tricuspid annular plane systolic excursion. HR of ANGPT2 and OPN represent an increase of one standard deviation.

Table 4: Prediction of mortality by ANGPT2 and TRAIL in multivariable cox regression.

\begin{tabular}{|c|c|c|c|c|c|c|c|}
\hline \multicolumn{8}{|c|}{ Multivariable cox regression on mortality } \\
\hline & $\mathrm{HR}$ & $95 \% \mathrm{Cl}$ & $\mathrm{p}$-value & & $\mathrm{HR}$ & $95 \% \mathrm{Cl}$ & $\mathrm{p}$-value \\
\hline ANGPT2 & 1.33 & $1.07-1.67$ & 0.012 & TRAIL & 0.66 & 0.44-0.99 & 0.045 \\
\hline Age & 1.07 & $1.04-1.10$ & $<0.001$ & Age & 1.07 & $1.04-1.09$ & $<0.001$ \\
\hline Male sex & 1.18 & $0.63-2.22$ & 0.598 & Male sex & 1.23 & $0.67-2.25$ & 0.510 \\
\hline mRSS & 1.05 & $1.02-1.08$ & $<0.001$ & mRSS & 1.04 & $1.01-1.06$ & 0.005 \\
\hline NT-proBNP & 1.01 & $1.01-1.01$ & $<0.001$ & NT-proBNP & 1.01 & $1.01-1.01$ & $<0.001$ \\
\hline Precapillary PH & 2.54 & $1.44-4.48$ & 0.001 & Precapillary PH & 2.03 & $1.19-3.46$ & 0.009 \\
\hline C-index & 0.83 & & & C-index & 0.82 & & \\
\hline
\end{tabular}

ANGPT2, angiopoietin 2; Cl, confidence interval; HR, hazard ratio; mRSS, modified Rodnan skin score; NTproBNP, N-terminal prohormone of brain natriuretic peptide; $\mathrm{PH}$, pulmonary hypertension; TRAIL, tumor 
necrosis factor-related apoptosis-inducing ligand. The HR of ANGPT2 and TRAIL represent an increase of one standard deviation.

\subsection{Serum markers and relation to pulmonary disease}

By logistic regression, neither OPN nor TRAIL showed association to ILD. OPN and TRAIL showed no correlation with mPAP, PVR, cardiac output (CO) nor cardiac index (CI). Angiopoietin showed a numerical association with ILD $(p=0.16)$ and a weak correlation with mPAP, PVR and $\mathrm{Cl}$, but not with $\mathrm{CO}$.

\section{Discussion}

LV and RV systolic and diastolic dysfunction are frequent cardiac complications of SSc and are associated with substantial reduced survival. Today, cardiac involvement often remains subclinical until severe organ manifestation is evident, and is often diagnosed at an advanced disease stage. Biomarkers revealing patients at high risk of cardiac dysfunction and increased mortality are scarce to date and therefore in demand $(9,10)$.

In the present study, we investigated candidate serum markers from two custom-tailored large panels for cardiopulmonary disease as a proof of concept study. We identified several markers associated with SSc in general, and several associated with cardiac disease. Our findings show an independent association of ANGPT2 with LV and RV systolic dysfunction, and mortality strongly linking this molecule to cardiac dysfunction in SSc. In addition, OPN, endostatin and TRAIL were associated with cardiac dysfunction, and notably, TRAIL was associated with diastolic dysfunction as well as mortality.

ANGPT2, OPN and TRAIL have all been previously shown altered in SSc. In addition, they have been reported altered in general cardiac disease $(40,42,43)$. These markers were therefore of particular interest for evaluation of cardiac disease associated with SSc. To our knowledge, this is the first study to present data on the association between biomarkers and cardiac dysfunction in SSc, potentially also representing novel targets for therapy in these patients. Here we show strong associations between these three circulating markers with LV and/or RV systolic dysfunction, and for TRAIL also with LV diastolic dysfunction. The three markers were also independently associated to mortality. In addition to prognostic and predictive potential, a greater understanding of these serum markers' physiologic characteristics may provide insight into the insufficiently comprehended pathophysiology of SSc. We do not expect these markers to replace the well appreciated NT-proBNP, and we have therefore neither fully investigated their association to NT-proBNP. However, this work is considered to be hypothesis generating, with a potential to enlighten and expand our knowledge on cardiac SSc. Given the retrospective nature of this study, it was not possible to relate the aforementioned markers to clinical cardiac symptoms.

ANGPT2 is produced by endothelial cells (44) and presumed to impair angiogenesis and promote cardiac fibrosis (42). ANGPT2 is an antagonist of ANGPT1, an anti-inflammatory protein stimulating vascular integrity and homeostasis (44). Our study supports earlier reports on higher serum concentrations of ANGPT2 in SSc patients in general, but without assessment to cardiac dysfunction (45). ANGPT2 has 
been suggested a potential therapeutic target against pathologic angiogenesis and inflammation (44). It is unclear whether ANGPT2 contributes to SSc pathophysiology or whether increased levels may reflect cardiac tissue at distress, or both. Future studies on whether ANGPT2 may predict cardiac disease, and whether regulation of ANGPT2 expression may have a therapeutic potential, are in demand.

TRAIL is a cytokine inducing endothelial cell apoptosis and smooth muscle proliferation (26). Serum levels are shown reduced in rheumatoid arthritis patients with heart failure (46). Among patients with acute coronary syndrome, low TRAIL concentration is reported a strong predictor of poor prognosis (43). Further, it is suggested that inhibition of TRAIL may reverse PH (26). While our cohort presented reduced levels of TRAIL in cardiac SSc, an earlier small scale study $(n=30)$ reported increased serum values of TRAIL among SSc patients compared to healthy controls, with no relation to cardiac involvement (47). Different results may also reflect dual effects of apoptosis. Thus, while apoptosis could attenuate inflammation at an early stage of disease, it could enhance cardiac failure in a fibrotic myocardium.

OPN is a cytokine recruiting macrophages and T-cells to site of inflammation. In rodents, OPN is reported upregulated in cardiac fibrosis (24) and to promote collagen synthesis (48), possibly at least at an early stage protecting the LV from dilation and systolic dysfunction. OPN has further been suggested as a therapeutic target (40). These findings are in concordance with our data, showing higher values in patients with reduced LV and/or RV systolic function. One may speculate whether regulation of OPN may reduce the burden of cardiac and/or pulmonary fibrosis in SSc. Future studies on the therapeutic potential of OPN regulation are in demand.

ANGPT2, OPN and TRAIL were all also strong predictors of mortality in our SSc cohort; even when adjusting for general risk factors of SSc. NT-proBNP lost significance when either ANGPT2 or OPN were included in multivariable model A including age, sex, diffusion capacity of the lungs, modified Rodnan skin score, NT-proBNP, LV diastolic dysfunction and RV systolic function. NT-proBNP is a highly recognized serum marker of heart failure, extensively utilized in clinical cardiology. NT-proBNP levels increase both with elevated cardiac pressures and reduced renal filtration. This may suggest a cardiac, or possibly renal, impact of ANGPT2 and OPN. As these two markers excluded NT-proBNP from prediction Model A, one may speculate whether these markers reveal cardiac, or non-cardiac, relations to mortality, superior to the ability of NT-proBNP. The roles of ANGPT2 and OPN are therefore especially interesting and call for future research approaches. The focus of this study is however not to design a prediction model for primary cardiac affection from SSc, but to cast light on novel promising serum markers.

Echocardiographic findings of cardiac dysfunction may be due to primary cardiac affliction, non-SSC general cardiac disease or $\mathrm{PH}$, the most feared complication of SSc. Increased pulmonary pressures increase RV pressure and may cause septal shift towards the LV, impairing LV filling during diastole. While the reported associations may reflect primary cardiac dysfunction due to SS, we cannot disregard that some of these associations may alternatively reflect precapillary PH. However, in this report, we show ANGPT2 and TRAIL to be independent predictors of mortality, adjusted for precapillary PH in multivariable analyses. Further, severe diastolic dysfunction is a very common cause of $\mathrm{PH}$ in the general 
population. Reports indicate that a considerable amount of patients with $\mathrm{PH}$ due to diastolic dysfunction may be misclassified as precapillary $\mathrm{PH}(49,50)$, enlightening some of the association between diastolic dysfunction and precapillary $\mathrm{PH}$. It is therefore of vital importance to evaluate the impact of cardiac SSc on mortality. We were not able to compare levels of the aforementioned serum markers between pre- and postcapillary $\mathrm{PH}$ due to a low number of patients with postcapillary $\mathrm{PH}$, which did not allow for meaningful statistical analyses.

Our study possesses some limitations worth recognizing. SSc patients may present cardiac dysfunction from non-SSc etiologies, which may dilute the characteristics of SSc-related cardiac dysfunction. Second, as echocardiographies were recorded from 2003, novel parameters as GLS were only evaluable for half of the cohort. Third, the nature of our study did not allow simultaneous serum sampling and echocardiography. In order to assure a temporal relation, only patients with a maximum of three years between echocardiography and sampling were included in analyses on GLS, diastolic dysfunction and TAPSE. Fourth, patients and controls were not completely matched to age and sex, which might have had an effect on serum marker levels. Last, while we consider our well-examined cohort favorable for evaluation of biomarker associations, we lack a validation cohort and validation studies are in demand.

The study also possesses significant strengths. This proof of concept serum marker study is to our knowledge the first study on serum markers of echocardiographic verified cardiac dysfunction in SSc. The study includes data on multiple parameters of a well-characterized cohort. Both panels were custom tailored for specific circulating markers known from cardiopulmonary disease. The levels were compared to a large control group of healthy individuals, analyzed at the time of patient serum analysis. Third, while the rarity of echocardiographic abnormalities limits the number of outcomes for analyzation purposes, the large size of our cohort allowed us to evaluate independent associations between serum markers and cardiac dysfunction using multivariable regression analyses.

\section{Conclusion}

In this study, we have shown ANGPT2, endostatin, OPN and TRAIL to be altered in SSc patients with cardiac dysfunction. ANGPT2, OPN and TRAIL were further strong independent predictors of mortality in combination with known risk factors for mortality in SSc. These markers may help enlighten the inadequately understood pathomechanisms of cardiac SSc, and could even potentially prove valuable for the diagnosis and treatment of this detrimental disease.

\section{Abbreviations}

ANGPT2, Angiopoietin 2; GLS, global longitudinal strain; PAH, pulmonary arterial hypertension; PH-ILD, pulmonary hypertension due to interstitial lung disease; OPN, osteopontin; TAPSE, tricuspid annular plane systolic excursion; TRAIL, tumor necrosis factor-related apoptosis-inducing ligand.

\section{Declarations}


- Ethics approval and consent to participate: All patients provided consent to participate in the study. The study was approved by The Regional Committee of Health and Medical Research Ethics in South-East Norway.

- Consent for publication: Not applicable.

- Availability of data and materials: The datasets used during the current study are available from the corresponding author on reasonable request.

- Competing interest:

- AH Tennøe has received consulting fees or other remuneration from GSK and Actelion.

- H Fretheim has received consulting fees or other remuneration from GSK and Actelion.

- H Didriksen has received consulting fees or other remuneration from GSK and Actelion.

- J A Belperio has received research funding and/or consulting fees or other remuneration from Boehringer Ingelheim and Genentech.

- AM Hoffmann-Vold has received research funding and/or consulting fees and/or other remuneration from Actelion, Boehringer Ingelheim, Roche, Janssen, Bayer, Merck Sharp \& Dohme, ARXX, Lilly, Medscape

The authors declare that they have no other competing interests.

- Funding: This work was supported by the Dam Foundation, Oslo, Norway, the Norwegian Women's Public Health Association, Oslo, Norway and the National Institutes of Health, Bethesda, Maryland, USA (Grant P01-HL108793). The funder was not included in analyzing data or writing of the manuscript.

- Author's contributions:

AHT participated in data collection, analyzing data and design/writing of the manuscript. KM participated in design of the manuscript. HD participated in data collection and analyzing data. TU participated in data collection. VP participated in data collection. SS participated in data collection. HF participated in data collection. ØMi participated in data collection. TG participated in data collection. CB participated in analyzing data. PA participated in data collection. ØM participated in design/writing of the manuscript. JB participated in data collection. AMHV participated in data collection, analyzing data and design/writing of the manuscript. All authors read and approved the final manuscript.

- Acknowledgements: Not applicable.

\section{References}

1. Pattanaik D, Brown M, Postlethwaite BC, Postlethwaite AE. Pathogenesis of Systemic Sclerosis. Front Immunol. 2015;6:272. 
2. Fretheim H, Halse AK, Seip M, Bitter H, Wallenius M, Garen T, et al. Multidimensional tracking of phenotypes and organ involvement in a complete nationwide systemic sclerosis cohort. Rheumatology (Oxford). 2020.

3. Elhai M, Meune C, Boubaya M, Avouac J, Hachulla E, Balbir-Gurman A, et al. Mapping and predicting mortality from systemic sclerosis. Ann Rheum Dis. 2017;76(11):1897-905.

4. Hoffmann-Vold AM, Fretheim H, Halse AK, Seip M, Bitter $H$, Wallenius $M$, et al. Tracking Impact of Interstitial Lung Disease in Systemic Sclerosis in a Complete Nationwide Cohort. Am J Respir Crit Care Med. 2019.

5. Xanthouli P, Jordan S, Milde N, Marra A, Blank N, Egenlauf B, et al. Haemodynamic phenotypes and survival in patients with systemic sclerosis: the impact of the new definition of pulmonary arterial hypertension. Ann Rheum Dis. 2020;79(3):370-8.

6. Perelas A, Silver RM, Arrossi AV, Highland KB. Systemic sclerosis-associated interstitial lung disease. Lancet Respir Med. 2020;8(3):304-20.

7. Allanore $Y$, Meune $C$. Primary myocardial involvement in systemic sclerosis: evidence for a microvascular origin. Clin Exp Rheumatol. 2010;28(5 Suppl 62):S48-53.

8. Pieroni M, De Santis M, Zizzo G, Bosello S, Smaldone C, Campioni M, et al. Recognizing and treating myocarditis in recent-onset systemic sclerosis heart disease: potential utility of immunosuppressive therapy in cardiac damage progression. Semin Arthritis Rheum. 2014;43(4):526-35.

9. Tennoe AH, Murbraech K, Andreassen JC, Fretheim H, Garen T, Gude E, et al. Left Ventricular Diastolic Dysfunction Predicts Mortality in Patients With Systemic Sclerosis. J Am Coll Cardiol. 2018;72(15):1804-13.

10. Tennøe AH, Murbræch K, Andreassen JC, Fretheim H, Midtvedt $\emptyset$, Garen T, et al. Systolic Dysfunction in Systemic Sclerosis: Prevalence and Prognostic Implications.0(0).

11. Meune C, Allanore Y, Devaux JY, Dessault O, Duboc D, Weber S, et al. High prevalence of right ventricular systolic dysfunction in early systemic sclerosis. J Rheumatol. 2004;31(10):1941-5.

12. Galie N, Humbert M, Vachiery JL, Gibbs S, Lang I, Torbicki A, et al. 2015 ESC/ERS Guidelines for the diagnosis and treatment of pulmonary hypertension: The Joint Task Force for the Diagnosis and Treatment of Pulmonary Hypertension of the European Society of Cardiology (ESC) and the European Respiratory Society (ERS): Endorsed by: Association for European Paediatric and Congenital Cardiology (AEPC), International Society for Heart and Lung Transplantation (ISHLT). Eur Heart J. 2016;37(1):67-119.

13. McLaughlin VV, Archer SL, Badesch DB, Barst RJ, Farber HW, Lindner JR, et al. ACCF/AHA 2009 expert consensus document on pulmonary hypertension a report of the American College of Cardiology Foundation Task Force on Expert Consensus Documents and the American Heart Association developed in collaboration with the American College of Chest Physicians; American Thoracic Society, Inc.; and the Pulmonary Hypertension Association. J Am Coll Cardiol. 2009;53(17):1573-619. 
14. Coghlan JG, Denton CP, Grunig E, Bonderman D, Distler O, Khanna D, et al. Evidence-based detection of pulmonary arterial hypertension in systemic sclerosis: the DETECT study. Ann Rheum Dis. 2014;73(7):1340-9.

15. Januzzi JL, van Kimmenade R, Lainchbury J, Bayes-Genis A, Ordonez-Llanos J, Santalo-Bel M, et al. NT-proBNP testing for diagnosis and short-term prognosis in acute destabilized heart failure: an international pooled analysis of 1256 patients: the International Collaborative of NT-proBNP Study. Eur Heart J. 2006;27(3):330-7.

16. Avouac J, Meune C, Chenevier-Gobeaux C, Borderie D, Lefevre G, Kahan A, et al. Cardiac biomarkers in systemic sclerosis: contribution of high-sensitivity cardiac troponin in addition to N-terminal probrain natriuretic peptide. Arthritis Care Res (Hoboken). 2015;67(7):1022-30.

17. Carulli MT, Handler C, Coghlan JG, Black CM, Denton CP. Can CCL2 serum levels be used in risk stratification or to monitor treatment response in systemic sclerosis? Ann Rheum Dis. 2008;67(1):105-9.

18. Schioppo T, Artusi C, Ciavarella T, Ingegnoli F, Murgo A, Zeni S, et al. N-TproBNP as biomarker in systemic sclerosis. Clin Rev Allergy Immunol. 2012;43(3):292-301.

19. Elhai M, Hoffmann-Vold AM, Avouac J, Pezet S, Cauvet A, Leblond A, et al. Performance of Candidate Serum Biomarkers for Systemic Sclerosis-Associated Interstitial Lung Disease. Arthritis Rheumatol. 2019;71(6):972-82.

20. Califf RM. Biomarker definitions and their applications. Exp Biol Med (Maywood). 2018;243(3):21321.

21. Hoffmann-Vold AM, Hesselstrand R, Fretheim H, Ueland T, Andreassen AK, Brunborg C, et al. CCL21 as a potential serum biomarker for pulmonary arterial hypertension in Systemic Sclerosis. Arthritis Rheumatol. 2018.

22. Reiseter S, Molberg O, Gunnarsson R, Lund MB, Aalokken TM, Aukrust P, et al. Associations between circulating endostatin levels and vascular organ damage in systemic sclerosis and mixed connective tissue disease: an observational study. Arthritis Res Ther. 2015;17:231.

23. Didriksen H FH, Palchevskiy V, Andreassen AK, Garen T, Midtvedt O, Gude E, Belperio JA, Molberg $\emptyset$, Hoffmann-Vold AM. The Lymphangiogenetic Factors VEGF-C, CCL21 and Ang-2 Are Associated with Pulmonary Arterial Hypertension in Systemic Sclerosis [abstract]. Arthritis Rheumatol 2018; 70 (suppl 10). 2018.

24. Matsui Y, Jia N, Okamoto H, Kon S, Onozuka H, Akino M, et al. Role of osteopontin in cardiac fibrosis and remodeling in angiotensin II-induced cardiac hypertrophy. Hypertension. 2004;43(6):1195-201.

25. Liu H, Yang E, Lu X, Zuo C, He Y, Jia D, et al. Serum levels of tumor necrosis factor-related apoptosisinducing ligand correlate with the severity of pulmonary hypertension. Pulm Pharmacol Ther. 2015;33:39-46.

26. Hameed AG, Arnold ND, Chamberlain J, Pickworth JA, Paiva C, Dawson S, et al. Inhibition of tumor necrosis factor-related apoptosis-inducing ligand (TRAIL) reverses experimental pulmonary hypertension. J Exp Med. 2012;209(11):1919-35. 
27. Preliminary criteria for the classification of systemic sclerosis (scleroderma). Subcommittee for scleroderma criteria of the American Rheumatism Association Diagnostic and Therapeutic Criteria Committee. Arthritis Rheum. 1980;23(5):581-90.

28. van den Hoogen F, Khanna D, Fransen J, Johnson SR, Baron M, Tyndall A, et al. 2013 classification criteria for systemic sclerosis: an American College of Rheumatology/European League against Rheumatism collaborative initiative. Arthritis Rheum. 2013;65(11):2737-47.

29. LeRoy EC, Black C, Fleischmajer R, Jablonska S, Krieg T, Medsger TA, Jr., et al. Scleroderma (systemic sclerosis): classification, subsets and pathogenesis. J Rheumatol. 1988;15(2):202-5.

30. Beyer C, Distler JH, Allanore Y, Aringer M, Avouac J, Czirjak L, et al. EUSTAR biobanking: recommendations for the collection, storage and distribution of biospecimens in scleroderma research. Ann Rheum Dis. 2011;70(7):1178-82.

31. Lemieux C, Maliba R, Favier J, Theoret JF, Merhi Y, Sirois MG. Angiopoietins can directly activate endothelial cells and neutrophils to promote proinflammatory responses. Blood. 2005;105(4):152330 .

32. Secchiero P, Gonelli A, Carnevale E, Milani D, Pandolfi A, Zella D, et al. TRAIL promotes the survival and proliferation of primary human vascular endothelial cells by activating the Akt and ERK pathways. Circulation. 2003;107(17):2250-6.

33. Hoffmann-Vold AM, Tennoe AH, Garen T, Midtvedt O, Abraityte A, Aalokken TM, et al. High Level of Chemokine CCL18 Is Associated With Pulmonary Function Deterioration, Lung Fibrosis Progression, and Reduced Survival in Systemic Sclerosis. Chest. 2016;150(2):299-306.

34. Lang RM, Badano LP, Mor-Avi V, Afilalo J, Armstrong A, Ernande L, et al. Recommendations for cardiac chamber quantification by echocardiography in adults: an update from the American Society of Echocardiography and the European Association of Cardiovascular Imaging. Eur Heart $\mathrm{J}$ Cardiovasc Imaging. 2015;16(3):233-70.

35. Takigiku K, Takeuchi M, Izumi C, Yuda S, Sakata K, Ohte N, et al. Normal range of left ventricular 2dimensional strain: Japanese Ultrasound Speckle Tracking of the Left Ventricle (JUSTICE) study. Circ J. 2012;76(11):2623-32.

36. Nagueh SF, Smiseth OA, Appleton CP, Byrd BF, 3rd, Dokainish H, Edvardsen T, et al. Recommendations for the Evaluation of Left Ventricular Diastolic Function by Echocardiography: An Update from the American Society of Echocardiography and the European Association of Cardiovascular Imaging. J Am Soc Echocardiogr. 2016;29(4):277-314.

37. Hoffmann-Vold AM, Fretheim H, Midtvedt O, Kilian K, Angelshaug M, Chaudhary A, et al. Frequencies of borderline pulmonary hypertension before and after the DETECT algorithm: results from a prospective systemic sclerosis cohort. Rheumatology (Oxford). 2017.

38. Simonneau G, Montani D, Celermajer DS, Denton CP, Gatzoulis MA, Krowka M, et al. Haemodynamic definitions and updated clinical classification of pulmonary hypertension. Eur Respir J. 2019;53(1).

39. Hoffmann-Vold AM, Aalokken TM, Lund MB, Garen T, Midtvedt O, Brunborg C, et al. Predictive value of serial high-resolution computed tomography analyses and concurrent lung function tests in 
systemic sclerosis. Arthritis Rheumatol. 2015;67(8):2205-12.

40. Waller AH, Sanchez-Ross M, Kaluski E, Klapholz M. Osteopontin in cardiovascular disease: a potential therapeutic target. Cardiol Rev. 2010;18(3):125-31.

41. Ueland T, Aukrust P, Nymo SH, Kjekshus J, McMurray JJ, Wikstrand J, et al. Predictive value of endostatin in chronic heart failure patients with poor kidney function. Cardiology. 2015;130(1):1722.

42. Chen JX, Zeng H, Reese J, Aschner JL, Meyrick B. Overexpression of angiopoietin-2 impairs myocardial angiogenesis and exacerbates cardiac fibrosis in the diabetic $\mathrm{db} / \mathrm{db}$ mouse model. Am J Physiol Heart Circ Physiol. 2012;302(4):H1003-12.

43. Osmancik P, Teringova E, Tousek P, Paulu P, Widimsky P. Prognostic value of TNF-related apoptosis inducing ligand (TRAIL) in acute coronary syndrome patients. PLoS One. 2013;8(2):e53860.

44. Scholz A, Plate KH, Reiss Y. Angiopoietin-2: a multifaceted cytokine that functions in both angiogenesis and inflammation. Ann N Y Acad Sci. 2015;1347:45-51.

45. Dunne JV, Keen KJ, Van Eeden SF. Circulating angiopoietin and Tie-2 levels in systemic sclerosis. Rheumatol Int. 2013;33(2):475-84.

46. Dessein PH, Lopez-Mejias R, Ubilla B, Genre F, Corrales A, Hernandez JL, et al. TNF-related apoptosisinducing ligand and cardiovascular disease in rheumatoid arthritis. Clin Exp Rheumatol. 2015;33(4):491-7.

47. Azab NA, Rady HM, Marzouk SA. Elevated serum TRAIL levels in scleroderma patients and its possible association with pulmonary involvement. Clin Rheumatol. 2012;31(9):1359-64.

48. Trueblood NA, Xie Z, Communal C, Sam F, Ngoy S, Liaw L, et al. Exaggerated left ventricular dilation and reduced collagen deposition after myocardial infarction in mice lacking osteopontin. Circ Res. 2001;88(10):1080-7.

49. Fox BD, Shimony A, Langleben D, Hirsch A, Rudski L, Schlesinger R, et al. High prevalence of occult left heart disease in scleroderma-pulmonary hypertension. Eur Respir J. 2013;42(4):1083-91.

50. Halpern SD, Taichman DB. Misclassification of pulmonary hypertension due to reliance on pulmonary capillary wedge pressure rather than left ventricular end-diastolic pressure. Chest. 2009;136(1):37-43.

\section{Figures}




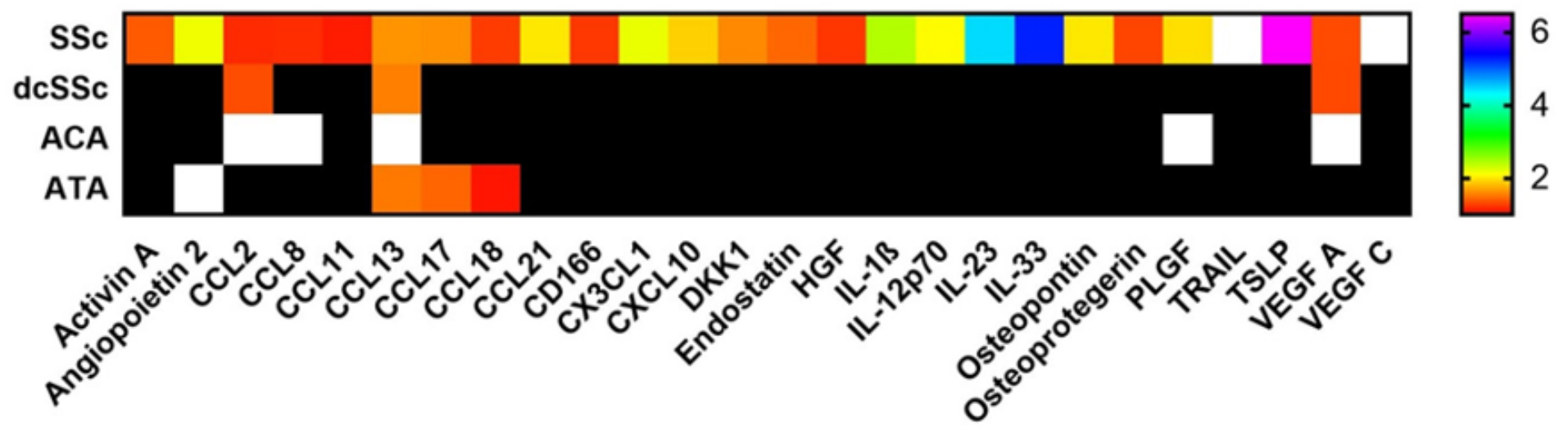

Figure 1

Heat map on circulating serum markers in SSc, diffuse SSc subset and SSc specific autoantibodies (Color print necessary)

Mean serum marker values of the SSc group compared to healthy controls and reported as color ratios. Subsets are compared within the SSc cohort; dcSSc is compared to IcSSc, while ACA and ATA positive subsets are compared to ACA and ATA negative subsets, respectively. Colors reflect upregulated serum marker ratios, white squares indicate downregulation, and black squares indicate no significant alteration. ACA, anti-centromere antibodies; ATA, anti-topoisomerase-I antibodies; CCL, C-C motif ligand; $\mathrm{CD}$, cluster of differentiation; $\mathrm{CXCL}, \mathrm{C}-\mathrm{X}-\mathrm{C}$ motif ligand; dcSSc, diffuse cutaneous systemic sclerosis; DKK1, Dickkopf-related protein 1; HGF, hepatocyte growth factor; IL, interleukin; PLGF, placental growth factor; SSc, systemic sclerosis; TRAIL, tumor necrosis factor-related apoptosis-inducing ligand; TSLP, thymic stromal lymphopoietin; VEGF, vascular endothelial growth factor. 


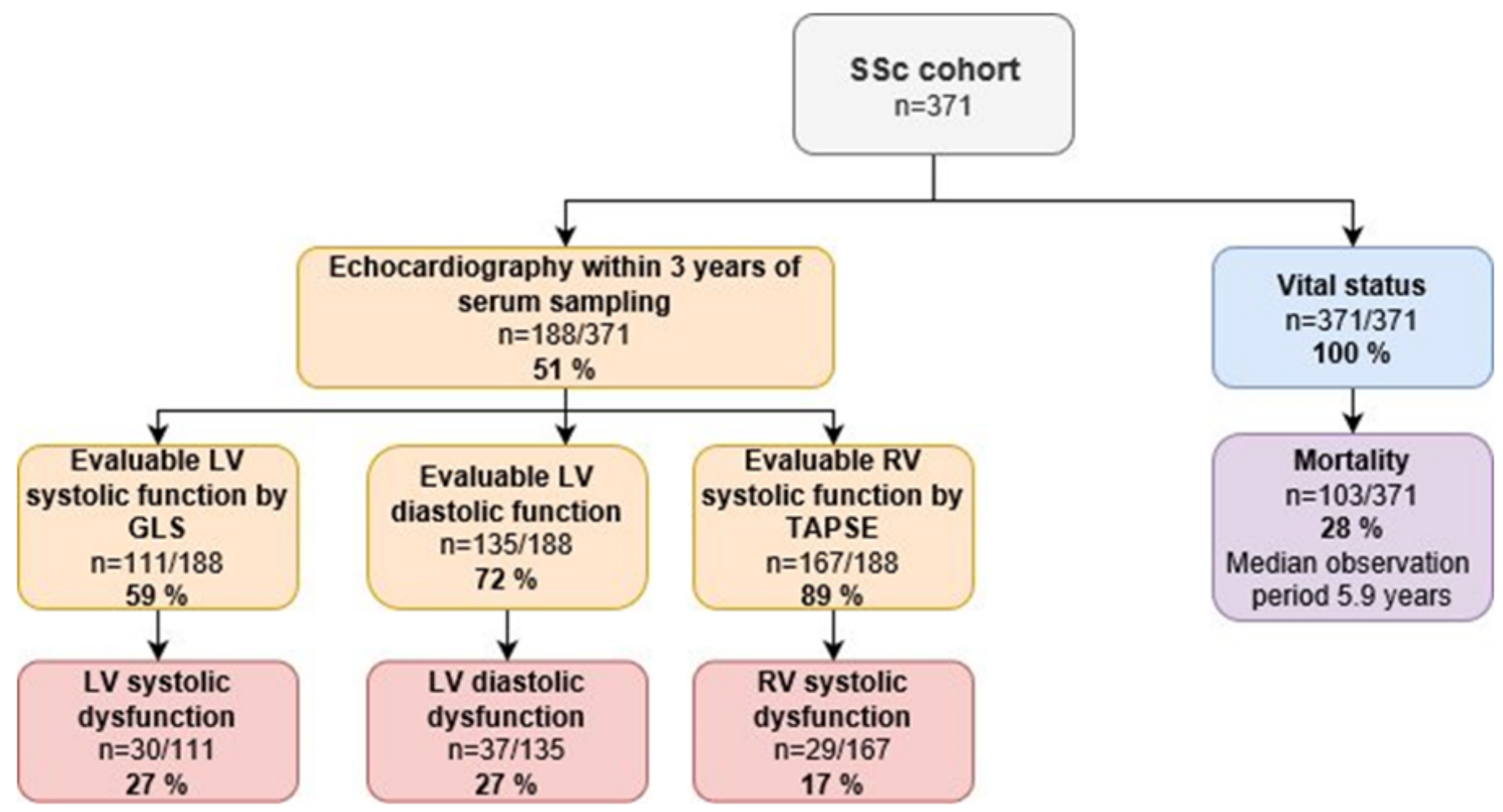

Figure 2

Flow chart on cardiac function and vital status of the SSc cohort.

GLS, global longitudinal strain; LV, left ventricular; RV, right ventricular; SSc, systemic sclerosis; TAPSE, tricuspid annular plane systolic excursion.

\section{Supplementary Files}

This is a list of supplementary files associated with this preprint. Click to download.

- DataSupplements.docx 